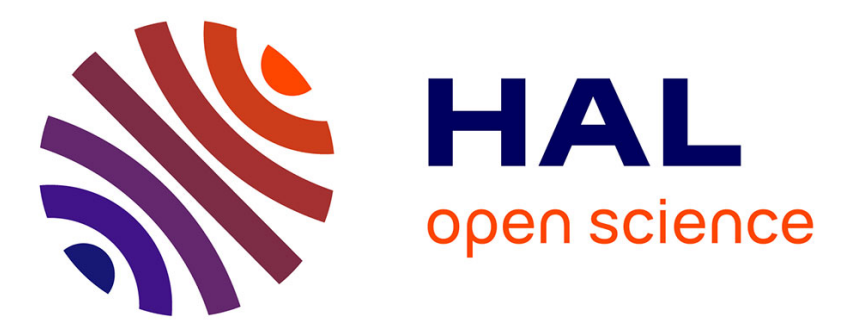

\title{
Some mixing properties of conditionally independent processes
}

Manel Kacem, Stéphane Loisel, Véronique Maume-Deschamps

\section{To cite this version:}

Manel Kacem, Stéphane Loisel, Véronique Maume-Deschamps. Some mixing properties of conditionally independent processes. Communications in Statistics - Theory and Methods, 2016, 45 (5), pp.1241-1259. hal-00670649

\section{HAL Id: hal-00670649 \\ https://hal.science/hal-00670649}

Submitted on 15 Feb 2012

HAL is a multi-disciplinary open access archive for the deposit and dissemination of scientific research documents, whether they are published or not. The documents may come from teaching and research institutions in France or abroad, or from public or private research centers.
L'archive ouverte pluridisciplinaire HAL, est destinée au dépôt et à la diffusion de documents scientifiques de niveau recherche, publiés ou non, émanant des établissements d'enseignement et de recherche français ou étrangers, des laboratoires publics ou privés. 


\title{
SOME MIXING PROPERTIES OF CONDITIONALLY INDEPENDENT PROCESSES
}

\author{
MANEL KACEM, STÉPHANE LOISEL, AND VÉRONIQUE MAUME-DESCHAMPS
}

\begin{abstract}
In this paper we consider conditionally independent processes with respect to some dynamic factor. More precisely, we assume that for all $i \in \mathbb{N}$, the random variables $X_{1}, \ldots, X_{i}$ are conditionally independent with respect to vector $\underline{V}_{i}=\left(V_{1}, \ldots, V_{i}\right)$. We study the mixing properties of the $X_{i}$ 's when conditioning is given with respect to unbounded memory of the factor. Our work is motivated by some real examples related to risk theory.
\end{abstract}

Acknowledgments. This work has been supported by the French Research National Agency (ANR) under reference ANR-08-BLAN-0314-01. The authors thank also the BNP Paribas Cardif Research Chair "Management de la modélisation" for financial support. The views expressed in this document are the authors' own and do not necessarily reflect those endorsed by BNP Paribas Cardif. The authors are also grateful to the Milliman Research Chair "Actuariat durable" for financial support.

\section{INTRODUCTION}

In risk theory the structure of dependence between individual risks may come from a time-varying common factor which represents the evolution of socio-economic and natural environment. Markov modulated models have been applied to risk theory for the first time by Asmussen (1989) [2] Cossette et al. (2002) 12] studied a Poisson risk model with common shock represented by a discrete random variable. More recently, Cossette et al. (2004) [13] proposed a compound binomial model modulated by a Markovian environment as an extension to the compound binomial model proposed by Gerber (1988) [22]. Denote by $\underline{V}_{n}$ the random vector $\left(V_{1}, \ldots, V_{n}\right)$ corresponding to the evolution of the factor up to time $n \geq 1$. Cossette et al. (2004) 13 considered a particular case of conditional independence: for any multi-indices $\left(i_{1}, \ldots, i_{u}\right), i_{1}<\cdots<i_{u}$ and any integer $n \geq i_{u}$,

$$
\mathbb{E}\left(f\left(X_{i_{1}}, \ldots, X_{i_{u}}\right) \mid \underline{V}_{n}\right)=\int_{x_{i_{1}}, \ldots, x_{i_{u}}} f\left(x_{i_{1}}, \ldots, x_{i_{u}}\right) \prod_{j=1}^{u} N^{i_{j}}\left(d x_{i_{j}}, V_{i_{j}}\right),
$$

where $N^{i}\left(d x_{i}, V_{i}\right)$ denotes the kernel transition of $X_{i}$ given $V_{i}$.

Due to claim settlement delays, accounting standards and risk time-accumulation phenomena, the whole memory of the factor often plays an important role in the risk process evolution in insurance. To our knowledge, conditionally

\footnotetext{
Key words and phrases. Conditional independence, risk processes, mixing properties.
} 
independent variables with respect to a random vector with time-varying length have not yet been considered in the literature. The aim of this paper is to extend the dependence frame introduced in Cossette et al. (2004) [13] by considering that dependence among risks may be explained by an unbounded memory of the common factor. This factor is not necessarily a Markov chain and may influence claim amounts (and) or frequencies. In this paper, we consider that r.v.'s $\left(X_{n}\right)_{n \in \mathbb{N}}$ are conditionally independent given the entire trajectory of the factor. More precisely, if we denote by $N^{j}\left(d x_{j}, \underline{V}_{j}\right)$ the kernel transition of $X_{j}$ given $\underline{V}_{j}$ we assume that for any multi-indices $\left(i_{1}, \ldots, i_{u}\right), i_{1}<\cdots<i_{u}$ and any integer $n \geq i_{u}$

$$
\mathbb{E}\left(f\left(X_{i_{1}}, \ldots, X_{i_{u}}\right) \mid \underline{V}_{n}\right)=\int_{x_{i_{1}}, \ldots, x_{i_{u}}} f\left(x_{i_{1}}, \ldots, x_{i_{u}}\right) \prod_{j=1}^{u} N^{i_{j}}\left(d x_{i_{j}}, \underline{V}_{i_{j}}\right) .
$$

Note that in this last case sequences $\left(\underline{V}_{j}\right)_{(j \in \mathbb{N})}$ overlap. We introduce our definition for mixing sequences and we derive some mixing properties for random processes subject to our time dependence model. Mixing processes have been initially studied by Rosenblatt (1956) [39], Kolmogorov and Rosanov (1960) 30, Ibragimov (1962) 26] and Ibragimov and Linnik (1971) 27]. Mixing condition for sequences of random variables breeds asymptotic independence between past and distant future events. This means that past is progressively forgotten. A simple way to check mixing conditions is to provide a precise control of the covariance as the distance between past and future goes to infinity or is sufficiently large. For a unified presentation of the main mixing coefficients and their properties we refer to Bradley and Bryc (1985) [7, Peligrad (1986) 34, Bradley $(1986,2005)$ [8, 9], Merlevède et al. (2006) [32] and Billingsley (1968) [5]. A large number of references concerning mixing coefficients and related dependence measures are given in Bradley (1986)[8] and Doukhan (1994)[19]. We follow the approach of Doukhan et al. (2007) [17] where the concepts and properties of weakly dependent processed are introduced.

It is well known that mixing properties are a key tool to derive moment conditions and asymptotic properties of aggregated processes. This is very important in economics and in actuarial science. In order to study asymptotic behavior of mixing sequences, one may either approximate mixing sequences with independent ones following Bernstein's method (1927) 4], or use Gordin's method (1969) [23] and approximate mixing sequences by a sequence of martingale differences (see e.g [24, 25, 35, 31] for further details).

The paper is organized as follows. In Section 2 we give a definition of mixing sequences thanks to a generalization of the one introduced in Doukhan and Louhichi (1999) 20 for weakly dependent r.v's., as well as the definitions of conditionally independent r.v's and conditionally strongly mixing sequences. Our main result is then stated in Theorem 2.2 in which we provide some mixing properties of the process $\left(X_{i}\right)_{i \in \mathbb{N}}$ under necessary conditions on the mixing properties of the factor process $\left(V_{i}\right)_{i \in \mathbb{N}}$ and on the conditional mixing structure. Section 3 is devoted to the proof of our main theorem. Section 4 deals with some examples that satisfy our assumptions. These examples are relevant in risk theory contexts. 


\section{Definitions and statement of Results.}

We first recall some standard definitions of mixing processes. Then we state our standing assumptions on conditional mixing and provide some basing properties. Finally, we state our main result (Theorem 2.2) which relates the mixing properties of conditionally independent processes to the mixing properties of the factor process and to the structure of the conditional independence.

2.1. Mixing sequences. Doukhan and Louhichi (1999)[20] introduced a unified weak dependence condition, more general than mixing condition, in which they make explicit the asymptotic independence between functions of the past and of the future of a sequence of random variable (and not between the past and the future of a sigma algebra as in Rosenblatt (1956) [39]).

Definition 1. Doukhan and Louhichi (1999) 20

The sequence $\left(X_{1}, \ldots, X_{n}\right)$ of r.v.s is called $(\theta, H, \psi)$ - weakly dependent, if there exist a class $H$ of real-valued functions, a sequence $\theta=\left(\theta_{r}\right)_{r \in \mathbb{N}}$ decreasing to 0 at infinity, and a function $\psi$ with arguments $(f, g, u, v) \in$ $H^{2} \times N^{2}$ such that for all $r \in \mathbb{N}$ and any

$i_{1} \leq \cdots \leq i_{u}<i_{u}+r \leq j_{1} \leq \cdots \leq j_{v}$ one has

$$
\left|\operatorname{Cov}\left(f\left(X_{i_{1}}, \ldots, X_{i_{u}}\right), g\left(X_{j_{1}}, \ldots, X_{j_{v}}\right)\right)\right| \leq \psi(f, g, u, v) \theta_{r},
$$

for all functions $f, g \in H$ that are defined respectively on $\mathbb{R}^{u}$ and $\mathbb{R}^{v}$. Note that $\theta$ depends on the class $H$ and on $\psi$. The function $\psi$ may depend on its argument which is not the case for classical strongly mixing sequences.

Note that if a sequence $\left(X_{1}, \ldots, X_{n}\right)$ is strongly mixing in the sense of Rosenblatt (1956) [39], then it is $\left(\theta, L^{\infty}, \psi\right)$ - weakly dependent where $L^{\infty}$ denotes the class of real-valued and bounded functions on some space of the form $\mathbb{R}^{p}$. Note that a variant of this notion of weak dependence is proposed in Coulon-Prieur and Doukhan (2000) [14. Following the definition introduced by Doukhan and Louhichi (1999) 20] we introduce the following definition.

Definition 2. Let $u, v$ be integers, a random process $\left(X_{1}, \ldots, X_{n}\right)$ is said to be $\eta_{u, v}$-mixing, if there exist a function $r \mapsto \eta(r)$ decreasing to 0 as $r$ goes to infinity and a constant $C(u, v)>0$ such that for any real valued bounded functions $f$ and $g$, for any integers $u^{\prime} \leq u, v^{\prime} \leq v$ and for any multi-indices satisfying the relation ( $)$ :

$$
i_{1}<\cdots<i_{u^{\prime}} \leq i_{u}<i_{u}+r \leq j_{1}<\cdots<j_{v^{\prime}} \leq j_{v},
$$

we have

$$
\sup \left|\operatorname{Cov}\left(f\left(X_{i_{1}}, \ldots, X_{i_{u^{\prime}}}\right), g\left(X_{j_{1}}, \ldots, X_{j_{v^{\prime}}}\right)\right)\right| \leq C(u, v) \eta(r)\|f\|_{a}\|g\|_{b},
$$

where the supremum is taken over all the sequences $\left(i_{1}, \ldots, i_{u^{\prime}}\right)$ and $\left(j_{1}, \ldots, j_{v^{\prime}}\right)$ satisfying ( () and $r \geq j_{1}-i_{u}$ is the gap of time between past and future. \|\|$_{a}$ and \|\|$_{b}$ are norms on bounded functions (or on subspaces of bounded functions). With respect to these norms, we have various kind of mixing as referred to the classical strongly mixing definitions.

(1) If \|\|$_{a}=\|\|_{b}=\|\|_{\infty}$, we shall say that the process is $\alpha_{(u, v)}$ mixing and we shall write $\alpha(r)$ instead of $\eta(r)$. If the process is $\alpha_{(u, v)}$ for all 
$u, v$ and if $\sup _{u, v \in \mathbb{N}} C(u, v) \leq C<\infty$ then the process is $\alpha$ mixing in the sense of Rosenblatt (1956)[39].

(2) If \|\|$_{a}=\|\|_{b}=\|\|_{2}$, we shall say that the process is $\rho_{(u, v)}$ mixing and we shall write $\rho(r)$ instead of $\eta(r)$. If the process is $\rho_{(u, v)}$ mixing for all $u, v$ and if $\sup _{u, v \in \mathbb{N}} C(u, v) \leq C<\infty$ then the process is $\rho$ mixing in the sense of Kolmogorov and Rozanov (1960)[30].

(3) If \|\|$_{a}=\|\|_{1}$ and \|\|$_{b}=\|\|_{\infty}$, we shall say that the process is $\Phi_{(u, v)}$ mixing and we shall write $\Phi(r)$ instead of $\eta(r)$. If the process is $\Phi_{(u, v)}$ mixing for all $u, v$ and if $\sup _{u, v \in \mathbb{N}} C(u, v) \leq C<\infty$ then the process is $\Phi$ mixing in the sense of Ibragimov (1962)[26].

(4) If \|\|$_{a}=\|\|_{b}=\|\|_{1}$, we shall say that the process is $\Psi_{(u, v)}$ mixing and we shall write $\Psi(r)$ instead of $\eta(r)$. If the process is $\Psi_{(u, v)}$ mixing for all $u, v$ and if $\sup _{u, v \in \mathbb{N}} C(u, v) \leq C<\infty$ then the process is $\Psi$ mixing in the sense of Blum et al. (1963) [6].

In the strongly mixing case, Davydov (1968)[15] first studied the problem of getting an upper bound for the covariance between two real-valued r.v's with given marginal distributions and given strong mixing coefficient. Rio (1993) [37] established a new covariance inequality improving Davydov's (1968) [15] one. It is well-known that every $\Psi$ mixing sequence is $\Phi$ mixing, every $\Phi$ mixing sequence is $\rho$ mixing and every $\rho$ mixing sequence is $\alpha$ mixing. The same proofs remain valid to get the chain

$$
\Psi_{(u, v)} \text { mixing } \Longrightarrow \Phi_{(u, v)} \text { mixing } \Longrightarrow \rho_{(u, v)} \text { mixing } \Longrightarrow \alpha_{(u, v)} \text { mixing. }
$$

Of course, sequences of independent random variables and $m$ - dependent sequences satisfy mixing conditions.

Various examples of processes satisfying mixing conditions are presented in the books of Doukhan (1994)[19] and Bradley (2007)[10].

Remark 1. For other choices of \|\|$_{a},\|\|_{b}$ (for example Lipschitz norms or bounded variation norms), we shall recover the notions of weak dependence (see [16, 18, 20, 17]). Note that for the classical mixing coefficients, $C(u, v)$ is uniform in $u$ and $v$.

Let us now introduce the main notions of conditional independence that we shall use.

2.2. Conditional independence. We shall make the following standing assumptions.

- H1: For all $i \in \mathbb{N}$, r.v's $X_{i}$ are conditionally independent given $\underline{V}_{i}=$ $\left(V_{1}, \ldots, V_{i}\right)$.

- H2: The conditional law of $X_{i} \mid \underline{V}_{n}$ is the same as the one of $X_{i} \mid \underline{V}_{i}$, for all $n \geq i$.

Assumptions $\mathrm{H} 1$ and $\mathrm{H} 2$ are equivalent to:

For any multi index $i_{1} \leq i_{2} \ldots \leq i_{u}$ and for any $n \geq i_{u}$ we have

$$
\mathbb{E}\left(f\left(X_{i_{1}}, \ldots, X_{i_{u}} \mid \underline{V}_{n}\right)\right)=\int_{x_{i_{1}}, \ldots, x_{i_{u}}} f\left(x_{i_{1}}, \ldots, x_{i_{u}}\right) \prod_{j=1}^{u} N^{i_{j}}\left(d x_{i_{j}}, \underline{V}_{i_{j}}\right),
$$

where $N^{i_{j}}\left(d x_{i_{j}}, \underline{V}_{i_{j}}\right)$ denotes the kernel transition of $X_{i_{j}}$ given $\underline{V}_{i_{j}}$. 
Proposition 2.1. Let $u, v$ and $r$ be integers and let $f$ and $g$ be real valued bounded functions. Consider the multi-indices $i_{1}<i_{2} \ldots<i_{u}<i_{u}+r \leq$ $j_{1}<j_{2} \ldots<j_{v}$. If $H 1$ and $H 2$ hold then

$$
\begin{aligned}
& \operatorname{Cov}\left(f\left(X_{i_{1}}, \ldots, X_{i_{u}}\right), g\left(X_{j_{1}}, \ldots, X_{j_{v}}\right)\right) \\
& \quad=\operatorname{Cov}\left(\mathbb{E}\left(f\left(X_{i_{1}}, \ldots, X_{i_{u}}\right) \mid \underline{V}_{i_{u}}\right), \mathbb{E}\left(g\left(X_{j_{1}}, \ldots, X_{j_{v}}\right) \mid \underline{V}_{j_{v}}\right)\right),
\end{aligned}
$$

This means that the covariance of $f\left(X_{i_{1}}, \ldots, X_{i_{u}}\right)$ and $g\left(X_{j_{1}}, \ldots, X_{j_{v}}\right)$ is equal to the covariance of their conditional expectation whenever conditioning is done respectively with respect to $\underline{V}_{i_{u}}$ and $\underline{V}_{j_{v}}$.

Proof. Denote by $X_{i_{1}}^{i_{u}}=\left(X_{i_{1}}, \ldots, X_{i_{u}}\right)$ and by $X_{j_{1}}^{j_{v}}=\left(X_{j_{1}}, \ldots, X_{j_{v}}\right)$. For any multi-indices $i_{1}<i_{2}<\ldots<i_{u}<j_{1}<j_{2}<\ldots<j_{v}$ and for any real valued functions $(f, g)$ for which the covariance function exists we have:

$$
\begin{aligned}
& \operatorname{Cov}\left(f\left(X_{i_{1}}^{i_{u}}\right), g\left(X_{j_{1}}^{j_{v}}\right)\right) \\
& =\mathbb{E}\left(f\left(X_{i_{1}}^{i_{u}}\right) \cdot g\left(X_{j_{1}}^{j_{v}}\right)\right)-\mathbb{E}\left(f\left(X_{i_{1}}^{i_{u}}\right)\right) \mathbb{E}\left(g\left(X_{j_{1}}^{j_{v}}\right)\right) \\
& =\mathbb{E}\left(\mathbb{E}\left(f\left(X_{i_{1}}^{i_{u}}\right) \mid \underline{V}_{i_{u}}\right) \cdot \mathbb{E}\left(g\left(X_{j_{1}}^{j_{v}}\right) \mid \underline{V}_{j_{v}}\right)\right)-\mathbb{E}\left\{\mathbb{E}\left(f\left(X_{i_{1}}^{i_{u}}\right) \mid \underline{V}_{i_{u}}\right)\right\} \mathbb{E}\left\{\mathbb{E}\left(g\left(X_{j_{1}}^{j_{v}}\right) \mid \underline{V}_{j_{v}}\right)\right\} \\
& =\operatorname{Cov}\left(\mathbb{E}\left(f\left(X_{i_{1}}^{i_{u}}\right) \mid \underline{V}_{i_{u}}\right), \mathbb{E}\left(g\left(X_{j_{1}}^{j_{v}}\right) \mid \underline{V}_{j_{v}}\right)\right) .
\end{aligned}
$$

2.3. Conditionally strongly mixing sequences given an unbounded memory. In our dependence framework we propose the following definition for conditionally strongly mixing sequences.

Definition 3. Let $u, v$ and $r$ be integers and let $\left(i_{1}, \ldots, i_{u}\right)$ and $\left(j_{1}, \ldots, j_{v}\right)$ satisfying ( $\star$ ) and let \|\|$_{a}$ and \|\|$_{b}$ be norms on bounded functions (or on subspaces of bounded functions).

The sequence of r.v's $\left(X_{n}\right)_{n \in \mathbb{N}}$ is called conditionally $\eta_{u, v}$ mixing with respect to $\underline{V}_{n}$ if there exist a positive sequence $\eta(r) \rightarrow 0$ as $r \rightarrow \infty$ and a constant $C(u, v)>0$ such that the following inequality holds for any real and bounded functions $f: \mathbb{R}^{u} \rightarrow \mathbb{R}$ and $g: \mathbb{R}^{v} \rightarrow \mathbb{R}$,

$$
\begin{aligned}
& \sup \left|\operatorname{Cov}\left(\mathbb{E}\left(f\left(X_{i_{1}}, \ldots, X_{i_{u^{\prime}}}\right) \mid \underline{V}_{i_{u^{\prime}}}\right), \mathbb{E}\left(g\left(X_{j_{1}}, \ldots, X_{j_{v^{\prime}}}\right) \mid \underline{V}_{j_{v^{\prime}}}\right)\right)\right| \\
& \leq C(u, v) \eta(r)\|f\|_{a}\|g\|_{b},
\end{aligned}
$$

where the supremum is taken over all sequences $\left(i_{1}, \ldots, i_{u^{\prime}}\right)$ and $\left(j_{1}, \ldots, j_{v^{\prime}}\right)$ satisfying (太).

Remark 2. Note that Definition 3 can be seen as a generalization of Definition 1 in Doukhan and Louhichi (1999)[20] by replacing in (2.5), $\underline{V}_{i_{u^{\prime}}}$ with $\underline{X}_{i_{1}}^{i_{u^{\prime}} \text { and }} \underline{V}_{j_{v^{\prime}}}$ with $\underline{X}_{j_{1}}^{j_{v^{\prime}}}$.

Remark 3. As for Definition 2, with respect to the choice of the norms \|\|$_{a}$ and \|\|$_{b}$, we shall say that the process is $\alpha_{u, v}, \rho_{u, v}, \Phi_{u, v}$ or $\Psi_{u, v}$ conditionally mixing with respect to $\left(\underline{V}_{n}\right)$.

Let us mention that the terminology conditionally mixing has been used with different meanings by Parakasa Rao (2009) [36], Dziubdziela (1986) [21] 
and Veijanen (1990) 40]. In particular, the work of Veijanen is concerned with partially observable random fields. The author considers that $\left(X_{i}, \ldots, X_{j}\right)$ is conditionally independent of $V_{r}, r \notin[i, j]$ given $\left(V_{i}, \ldots, V_{j}\right)$ for any positive integers $i$ and $j$, which is not our case here.

Below, we give an example showing that the conditional independence related to the whole vector $\underline{V}_{i}$ implies a dependence that may be persistent for the process $\left(X_{i}\right)_{i \in \mathbb{N}}$. In particular, our conditional independence condition and some mixing condition on the factor process $\left(V_{i}\right)_{i \in \mathbb{N}}$ are not sufficient to get mixing properties for the process $\left(X_{i}\right)_{i \in \mathbb{N}}$.

Example 1. Consider $Y_{i}=I_{i}, \forall i \geq 1, P\left(I_{i}=1 \mid \underline{V}_{i}\right)=\frac{1}{2}\left(V_{1}+V_{i}\right)$. Let $\left(V_{i}\right)_{i \in \mathbb{N}}$ be a sequence of identically distributed r.v's with $\mathbb{E}\left(V_{1}\right)=p$. Then we have $P\left(I_{i}=1\right)=\mathbb{E}\left(V_{1}\right)=p$ and $P\left(I_{r}=1\right)=\mathbb{E}\left(V_{1}\right)=p$. Let

$$
P\left(I_{1}=1, I_{r}=1 \mid V_{1}, V_{r}\right)=P\left(I_{1}=1 \mid V_{1}\right) P\left(I_{r}=1 \mid V_{1}, V_{r}\right)=\frac{1}{2} V_{1}\left(V_{1}+V_{r}\right) .
$$

Then we have $P\left(I_{1}=1, I_{r}=1\right)=\frac{1}{2} \mathbb{E}\left(V_{1} \times\left(V_{1}+V_{r}\right)\right)$. We thus get

$$
\begin{aligned}
P\left(I_{1}\right. & \left.=1, I_{r}=1\right)-P\left(I_{1}=1\right) P\left(I_{r}=1\right) \\
& =\frac{1}{2}\left(\mathbb{E}\left(V_{1}^{2}\right)+\mathbb{E}\left(V_{1} V_{r}\right)-\mathbb{E}\left(V_{1}\right)\left(\mathbb{E}\left(V_{1}\right)+\mathbb{E}\left(V_{r}\right)\right)\right) \\
& =\frac{1}{2}(\mathbb{E}\left(V_{1}^{2}\right)-\mathbb{E}\left(V_{1}\right)^{2}+\underbrace{\mathbb{E}\left(V_{1} V_{r}\right)-\mathbb{E}\left(V_{1}\right) \mathbb{E}\left(V_{r}\right)}_{(* *)}) .
\end{aligned}
$$

If the factor process $\left(V_{i}\right)_{i \in \mathbb{N}}$ is independent then $(* *)=0$ and then,

$$
P\left(I_{1}=1, I_{r}=1\right)-P\left(I_{1}=1\right) P\left(I_{r}=1\right)=\frac{1}{2} p(p-1)
$$

for all $r \in \mathbb{N}$.

Therefore, in this example assuming that sequence $\left(Y_{i}\right)$ satisfies conditional independence is not sufficient to have mixing condition. This is due to the long memory of the factor taken into account when conditioning.

Proposition 2.1 proves that if the process $\left(X_{i}\right)_{i \in \mathbb{N}}$ is conditionally independent and conditionally $\eta_{u, v}$ mixing with respect to $\left(V_{i}\right)_{i \in \mathbb{N}}$ then it is $\eta_{u, v}$ mixing, with the same kind of $\eta$ mixing.

2.4. Main result. Our main result emphasizes the relation between the conditional mixing properties of the process $\left(X_{i}\right)_{i \in \mathbb{N}}$, the mixing properties of the process $\left(V_{i}\right)_{i \in \mathbb{N}}$ and the regularity of the transition kernels. Recall Equation 1.2): if we denote by $N^{i_{1}, \ldots, i_{u}}\left(\cdot, \underline{V}_{i_{u}}\right)$ the kernel transition of the random vector $\left(X_{i_{1}}, \ldots, X_{i_{u}}\right)$ given $\underline{V}_{i_{u}}=\left(V_{1}, \ldots, V_{i_{u}}\right)$, then the conditional independence condition implies

$$
N^{i_{1}, \ldots, i_{u}}\left(\cdot, \underline{V}_{i_{u}}\right)=\prod_{k=1}^{u} N^{i_{k}}\left(\cdot, \underline{V}_{i_{k}}\right) .
$$

Consider multi indices $\left(i_{1}, \ldots, i_{u}\right)$ and $\left(j_{1}, \ldots, j_{v}\right)$, satisfying $(\star)$, let $\ell$ be an integer such that $0<\ell \leq j_{1}-i_{u}$. Let $\widetilde{V}_{i_{u}, j_{v}, \ell}=\left(0, \ldots, 0, V_{i_{u}+\ell+1}, \ldots, V_{j_{1}}, \ldots, V_{j_{v}}\right) \in$ $\mathbb{R}^{j_{v}}$ and denote

$$
D\left(\underline{V}_{j_{v}}, \widetilde{V}_{i_{u}, j_{v}, \ell}\right)=N^{j_{1}, \ldots, j_{v}}\left(\cdot, \underline{V}_{j_{v}}\right)-N^{j_{1}, \ldots, j_{v}}\left(\cdot, \widetilde{V}_{i_{u}, j_{v}, \ell}\right) .
$$


In what follows, given two measures $\nu$ and $\mu$ on the same $\sigma$-field, we shall say that $\nu \leq \mu$ if for any measurable set $A, \nu(A) \leq \mu(A)$ or equivalently, if for any non negative and bounded function $f, \nu(f) \leq \mu(f)$. We shall make the following assumptions on $D\left(\underline{V}_{j_{v}}, \widetilde{V}_{i_{u}, j_{v}, \ell}\right)$ and $N^{j_{1}, \ldots, j_{v}}$.

Assumption 1. $K_{v}$ and $K_{v}^{\prime}$ denote positive constants depending on $v$, $(\kappa(\ell))_{\ell \in \mathbb{N}}$ denotes a decreasing to zero sequence and $\widetilde{N}_{j_{1}, \ldots, j_{v}}(\cdot)$ is a positive finite measure on $\mathbb{R}^{v}$. We denote $k=j_{1}-i_{u}-\ell$ with $0<\ell \leq j_{1}-i_{u}$. Let us introduce the following assumptions satisfied for multi indices $\left(i_{1}, \ldots, i_{u}\right)$ and $\left(j_{1}, \ldots, j_{v}\right)$ satifying $\star$.

$$
\begin{gathered}
\sup _{\underline{V}}\left|D\left(\underline{V}_{j_{v}}, \widetilde{V}_{i_{u}, j_{v}, \ell}\right)\right| \leq K_{v}\left\|N^{j_{1}, \ldots, j_{v}}\left(\cdot, \underline{V}_{j_{v}}\right)\right\|_{1} \kappa(k) . \\
\sup _{\underline{V}}\left|D\left(\underline{V}_{j_{v}}, \widetilde{V}_{i_{u}, j_{v}, \ell}\right)\right| \leq \widetilde{N}_{j_{1}, \ldots, j_{v}}(\cdot) \kappa(k) . \\
\int_{\underline{V}} \int_{x_{j_{1}}, \ldots x_{j_{v}}}\left|D\left(\underline{V}_{j_{v}}, \widetilde{V}_{i_{u}, j_{v}, \ell}\right)\right| \leq \kappa(k) K_{v} . \\
\left\|N^{j_{1}, \ldots, j_{v}}\left(\cdot, \underline{V}_{j_{v}}\right)\right\|_{\infty} \leq K_{v}^{\prime}\left\|N^{j_{1}, \ldots, j_{v}}\left(\cdot, \underline{V}_{j_{v}}\right)\right\|_{1} . \\
\int_{x_{j_{1}}, \ldots x_{j_{v}}}\left\|N^{j_{1}, \ldots, j_{v}}\left(\cdot, \underline{V}_{j_{v}}\right)\right\|_{\infty}<\infty .
\end{gathered}
$$

Theorem 2.2. Assume $\left(X_{i}\right)_{i \in \mathbb{N}}$ is conditionally independent with respect to $\left(V_{i}\right)_{i \in \mathbb{N}}$.

\begin{tabular}{|c|c|c|}
\hline $\begin{array}{l}\text { Kind of mixing } \\
\text { for }\left(V_{i}\right)_{i \in \mathbb{N}}\end{array}$ & $\begin{array}{l}\text { Satisfied equation } \\
\text { of assumption } 1\end{array}$ & $\begin{array}{l}\text { Result: type of mixing } \\
\text { for }\left(X_{i}\right)_{i \in \mathbb{N}}\end{array}$ \\
\hline \multirow[t]{2}{*}{$\Psi_{u, v}$} & 2.6 & $\Psi_{u, v}$ \\
\hline & 2.7 & $\Phi_{u, v}$ \\
\hline$\Phi_{u, v}$ & (2.7) and (2.10) & $\Phi_{u, v}$ \\
\hline \multirow[t]{3}{*}{$\alpha_{u, v}$} & (2.6) and 2.9) & $\Psi_{u, v}$ \\
\hline & 2.8) and 2.9 & $\Phi_{u, v}$ \\
\hline & (2.8) and (2.10) & $\alpha_{u, v}$ \\
\hline
\end{tabular}

The results are presented in Table 1. As an example, one can read the first line of Table 1 as follows: if the process $\left(V_{i}\right)_{i \in \mathbb{N}}$ is $\Psi_{u, v}$ mixing and if (2.6) of Assumption 1 is satisfied then the process $\left(X_{i}\right)_{i \in \mathbb{N}}$ is $\Psi_{u, v}$-mixing.

\section{TABLE 1.}

It is worth pointing out that with some additional condition, the process $\left(X_{i}\right)_{i \in \mathbb{N}}$ may satisfy a stronger mixing property than that satisfied by the factor process $\left(V_{i}\right)_{i \in \mathbb{N}}$. For example, if the process $\left(V_{i}\right)_{i \in \mathbb{N}}$ is $\alpha_{u, v}$ mixing and (2.6) and (2.9) are satisfied then the process $\left(X_{i}\right)_{i \in \mathbb{N}}$ is $\Psi_{u, v}$ mixing. 


\section{Proof of the Main Result}

The proof of Theorem 2.2 is based on a decomposition of the covariance.

Lemma 3.1. Assume that $\left(V_{i}\right)_{i \in \mathbb{N}}$ is $\eta_{(u, v)}$ mixing in the sense of Definition 2. For any multi indices $i_{1}<\cdots<i_{u}<j_{1}<\cdots<j_{v}$ and $0<\ell \leq j_{1}-i_{u}$ and bounded functions $f$ and $g$, we have

$$
\begin{aligned}
& \left|\operatorname{Cov}\left(\mathbb{E}\left(f\left(X_{i_{1}}, \ldots, X_{i_{u}}\right) \mid \underline{V}_{i_{u}}\right), \mathbb{E}\left(g\left(X_{j_{1}}, \ldots, X_{j_{v}}\right) \mid \underline{V}_{j_{v}}\right)\right)\right| \\
& \leq 2\|f\|_{1} \int_{x_{j_{1}}, \ldots, x_{j_{v}}}|g| \cdot \sup _{\underline{V}}\left|D\left(\underline{V}_{j_{v}}, \widetilde{V}_{i_{u}, j_{v}, \ell}\right)\right|+ \\
& \quad \int_{x_{i_{1}}, \ldots, x_{i_{u}}, x_{j_{1}}, \ldots, x_{j_{v}}}|f g| \eta_{u, v}(\ell)\left\|N^{i_{1}, \ldots, i_{u}}\left(\cdot, \underline{V}_{i_{u}}\right)\right\|_{a} \cdot\left\|N^{j_{1}, \ldots, j_{v}}\left(\cdot, \widetilde{V}_{i_{u}, j_{v}, \ell}\right)\right\|_{b} .
\end{aligned}
$$

Proof. We remark that

$$
\begin{aligned}
& \left|\operatorname{Cov}\left(\mathbb{E}\left(f\left(X_{i_{1}}, \ldots, X_{i_{u}}\right) \mid \underline{V}_{i_{u}}\right), \mathbb{E}\left(g\left(X_{j_{1}}, \ldots, X_{j_{v}}\right) \mid \underline{V}_{j_{v}}\right)\right)\right| \\
& =\left|\operatorname{Cov}\left(\int_{x_{i_{1}}, \ldots, x_{i_{u}}} f \cdot N^{i_{1}, \ldots, i_{u}}\left(\cdot, \underline{V}_{i_{u}}\right), \int_{x_{j_{1}}, \ldots, x_{j_{v}}} g \cdot N^{j_{1}, \ldots, j_{v}}\left(\cdot, \underline{V}_{j_{v}}\right)\right)\right| \\
& =\left|\begin{array}{c}
\int_{x_{i_{1}}, \ldots, x_{i_{u}}, x_{j_{1}}, \ldots, x_{j_{v}}} f g \cdot \operatorname{Cov}_{\underline{V}}\left(N^{i_{1}, \ldots, i_{u}}\left(\cdot, \underline{V}_{i_{u}}\right), N^{j_{1}, \ldots, j_{v}}\left(\cdot, \underline{V}_{j_{v}}\right)\right)
\end{array}\right|,
\end{aligned}
$$

and

$$
\begin{aligned}
\left|\operatorname{Cov}_{\underline{\underline{V}}}\left(N^{i_{1}, \ldots, i_{u}}\left(\cdot, \underline{V}_{i_{u}}\right), N^{j_{1}, \ldots, j_{v}}\left(\cdot, \underline{V}_{j_{v}}\right)\right)\right| \leq & \left|\operatorname{Cov}_{\underline{\underline{V}}}\left(N^{i_{1}, \ldots, i_{u}}\left(\cdot, \underline{V}_{i_{u}}\right), D\left(\underline{V}_{j_{v}}, \widetilde{V}_{i_{u}, j_{v}, \ell}\right)\right)\right| \\
& +\left|\operatorname{Cov}_{\underline{V}}\left(N^{i_{1}, \ldots, i_{u}}\left(\cdot, \underline{V}_{i_{u}}\right), N^{j_{1}, \ldots, j_{v}}\left(\cdot, \widetilde{V}_{i_{u}, j_{v}, \ell}\right)\right)\right| .
\end{aligned}
$$

Now, because $\left(V_{i}\right)_{i \in \mathbb{N}}$ satisfies the $\eta_{u, v}$ mixing property,

$\left|\operatorname{Cov}_{\underline{V}}\left(N^{i_{1}, \ldots, i_{u}}\left(\cdot, \underline{V}_{i_{u}}\right), N^{j_{1}, \ldots, j_{v}}\left(\cdot, \widetilde{V}_{i_{u}, j_{v}, \ell}\right)\right)\right| \leq \eta_{u, v}(\ell)\left\|N^{i_{1}, \ldots, i_{u}}\left(\cdot, \underline{V}_{i_{u}}\right)\right\|_{a}\left\|N^{j_{1}, \ldots, j_{v}}\left(\cdot, \widetilde{V}_{i_{u}, j_{v}, \ell}\right)\right\|_{b}$.

On the other hand,

$$
\int_{\underline{V}} \int_{x_{i_{1}}, \ldots, x_{i_{u}}}|f| \cdot N^{i_{1}, \ldots, i_{u}}\left(\cdot, \underline{V}_{i_{u}}\right) d \underline{V}=\|f\|_{1} .
$$

This concludes the proof.

Lemma 3.2. Assume that $\left(V_{i}\right)_{i \in \mathbb{N}}$ is $\eta_{(u, v)}$ mixing in the sense of Definition 2. For any multi indices $i_{1}<\cdots<i_{u}<j_{1}<\cdots<j_{v}$ and $0<\ell \leq j_{1}-i_{u}$ and bounded functions $f$ and $g$, we have

$$
\begin{aligned}
\left|\operatorname{Cov}\left(\mathbb{E}\left(f\left(X_{i_{1}}, \ldots, X_{i_{u}}\right) \mid \underline{V}_{i_{u}}\right), \mathbb{E}\left(g\left(X_{j_{1}}, \ldots, X_{j_{v}}\right) \mid \underline{V}_{j_{v}}\right)\right)\right| \\
\leq 2 \int_{x_{i_{1}}, \ldots, x_{i_{u}}}|f| \sup _{\underline{V}} N^{i_{1}, \ldots, i_{u}}\left(\cdot, \underline{V}_{i_{u}}\right) \times\|g\|_{\infty} \int_{\underline{V}} \int_{x_{j_{1}}, \ldots, x_{j_{v}}}\left|D\left(\underline{V}_{j_{v}}, \widetilde{V}_{i_{u}, j_{v}, \ell}\right)\right|+ \\
\quad \int_{x_{i_{1}}, \ldots, x_{i_{u}}, x_{j_{1}}, \ldots, x_{j_{v}}}|f g| \eta_{u, v}(\ell)\left\|N^{i_{1}, \ldots, i_{u}}\left(\cdot, \underline{V}_{i_{u}}\right)\right\|_{a} \cdot\left\|N^{j_{1}, \ldots, j_{v}}\left(\cdot, \widetilde{V}_{i_{u}, j_{v}, \ell}\right)\right\|_{b}
\end{aligned}
$$


Proof. The proof is done in the same way as the previous one.

Proof of Theorem 2.2. We shall give the complete proof of the fact that If $\left(V_{i}\right)_{i \in \mathbb{N}}$ is $\Psi_{u, v}$ mixing and 2.6) of Assumption 1 is satisfied then $\left(X_{i}\right)_{i \in \mathbb{N}}$ is $\Psi_{u, v}$-mixing.

The other cases are treated similarly. Note that when $(2.6)$ or $(2.7)$ are satisfied, we use Lemma 3.1 while when $(2.8)$ is fulfilled, we use Lemma 3.2 . From Proposition 2.1, in order to prove that $\left(X_{i}\right)_{i \in \mathbb{N}}$ is $\Psi_{u, v}$-mixing, it suffices to prove that it is conditionally $\Psi_{u, v}$-mixing with respect to $\left(V_{i}\right)_{i \in \mathbb{N}}$. Consider multi indices $\left(i_{1}, \ldots, i_{u}\right)$ and $\left(j_{1}, \ldots, j_{v}\right)$ satisfying $(\star)$. Let $r=$ $j_{1}-i_{u}$ and $0<\ell<r$. From Lemma 3.1, 2.6) of Assumption 1 and the $\Psi_{(u, v)}$ mixing of $\left(V_{i}\right)_{i \in \mathbb{N}}$, we have

$$
\begin{aligned}
& \left|\operatorname{Cov}\left(\mathbb{E}\left(f\left(X_{i_{1}}, \ldots, X_{i_{u}}\right) \mid \underline{V}_{i_{u}}\right), \mathbb{E}\left(g\left(X_{j_{1}}, \ldots, X_{j_{v}}\right) \mid \underline{V}_{j_{v}}\right)\right)\right| \\
& \left.\leq 2\|f\|_{1} \kappa(r-\ell) K_{v} \int_{x_{j_{1}}, \ldots, x_{j_{v}}} g \| N^{j_{1} \ldots x_{j_{v}}}\left(\cdot, \underline{V}_{j_{v}}\right)\right) \|_{1}+ \\
& \quad \int_{x_{i_{1}, \ldots, x_{i_{u}}, x_{j_{1}}, \ldots, x_{j_{v}}}} f g \Psi_{u, v}(\ell)\left\|N^{i_{1}, \ldots, i_{u}}\left(\cdot, \underline{V}_{i_{u}}\right)\right\|_{1} \cdot\left\|N^{j_{1} \ldots, j_{v}}\left(\cdot, \widetilde{V}_{i_{u}, j_{v}, \ell}\right)\right\|_{1} \\
& =2 K_{v} \kappa(r-\ell)\|f\|_{1}\|g\|_{1}+\Psi_{u, v}(\ell)\|f\|_{1}\|g\|_{1} .
\end{aligned}
$$

We choose $\ell=\left\lfloor\frac{r}{2}\right\rfloor+1$ to conclude.

\section{EXAMPLES AND SOME APPLICATIONS}

In this section, we provide some examples of conditionally independent random variables $\left(X_{n}\right)_{n \in \mathbb{N}}$ given a factor $\left(V_{1}, \ldots, V_{n}\right)$ that satisfy conditions of Theorem 2.2. First of all, we remark that if the conditional mixing has bounded memory - roughly speaking if the conditional law $X_{i} \mid \underline{V}$ is the same as $X_{i} \mid\left(V_{i}, \ldots, V_{i-\ell}\right)$ - then we derive strong mixing properties of $\left(X_{i}\right)_{i \in \mathbb{N}}$ from the mixing properties of $\left(V_{i}\right)_{i \in \mathbb{N}}$; additional properties on the structure of the conditioning are not required. Secondly, we provide examples with unbounded memory that satisfy Assumption 1 .

4.1. Mixing properties for sequence controlled by a bounded memory of the factor. Note that bounded memory means here memory of order $\ell \in \mathbb{N}$. In classical risk theory related with conditionally independent r.v's $\left(X_{n}\right)_{n \in \mathbb{N}}$ given a factor $\left(V_{1}, \ldots, V_{n}\right)$ it is very useful to consider that the conditional distribution of $X_{i}$ depends only on the value of $V_{i}$. This case corresponds to $\ell=0$ and is considered for example in Cossette et al. (2004) [13. In connection with mixing type of dependence the reader is referred to Obrein's (1974) 33 work where the factor is modulated by a Markov chain.

Next we give the following proposition satisfied for all $\ell \geq 0$.

Let $(u, v)$ be a couple of integers. Write $\underline{V}_{i, \ell}=\left(V_{i}, \ldots, V_{i-\ell}\right), \underline{V}_{i_{1}, i_{u}, \ell}=$ $\left(V_{i_{u}}, \ldots, V_{i_{1}-\ell}\right)$ and $\underline{V}_{j_{1}, j_{v}, \ell}=\left(V_{j_{v}}, \ldots, V_{j_{1}-\ell}\right)$.

Proposition 4.1. Let $\left(X_{i}\right)_{i \in N}$ be a sequence of conditionally independent random variables given $\left(\underline{V}_{i, \ell}\right)_{i \in N}$. Let $p, q \in[1, \infty)$, assume that $\left(V_{i}\right)_{i \in \mathbb{N}}$ is $\eta_{u, v}$ mixing with respect to the $L^{p}$ and $L^{q}$ norms, with mixing coefficient 
denoted by $\eta(r)$. Then $\left(X_{i}\right)_{i \in \mathbb{N}}$ satisfies also the $\eta_{u, v}$ mixing condition with respect to the $L^{p}$ and $L^{q}$ norms and the mixing coefficient is $\eta^{\prime}(r)=\eta(r-\ell)$.

Proof. With the notation

$$
\mathbb{E}\left(f\left(X_{i_{1}}, \ldots, X_{i_{u}}\right) \mid \underline{V}_{i_{1}, i_{u}, \ell}\right)=h_{1}\left(\underline{V}_{i_{1}, i_{u}, \ell}\right),
$$

and

$$
\mathbb{E}\left(g\left(X_{j_{1}}, \ldots, X_{j_{v}}\right) \mid \underline{V}_{j_{1}, j_{v}, \ell}\right)=h_{2}\left(\underline{V}_{j_{1}, j_{v}, \ell}\right),
$$

since $f, g$ are two bounded real functions, we have

$$
\begin{aligned}
& \left|\operatorname{Cov}\left(\mathbb{E}\left(f\left(X_{i_{1}}, \ldots, X_{i_{u}}\right) \mid \underline{V}_{i_{1}, i_{u}, \ell}\right), \mathbb{E}\left(g\left(X_{j_{1}}, \ldots, X_{j_{v}}\right) \mid \underline{V}_{j_{1}, j_{v}, \ell}\right)\right)\right| \\
& =\left|\operatorname{Cov}\left(h_{1}\left(\underline{V}_{i_{1}, i_{u}, \ell}\right), h_{2}\left(\underline{V}_{j_{1}, i_{v}, \ell}\right)\right)\right| \\
& \leq C(u, v) \eta(r-\ell)\left\|h_{1}\right\|_{p}\left\|h_{2}\right\|_{q} \leq C(u, v) \eta(r-\ell)\|f\|_{p}\|g\|_{q},
\end{aligned}
$$

using Jensen's inequality.

4.2. Mixing properties for sequence controlled by a factor with an unbounded memory. It should be noted that by unbounded memory of the factor we mean that the conditional independence is with respect to a length varying factor vector. Such type of conditioning has not been considered in the literature to our knowledge. In addition to this fact, our study is motivated by the interest of such type of conditioning in describing models with time dependent risks in finance and insurance. Indeed, we wish to compare the aggregate risk of an insurance portfolio of conditionally independent risks when the conditioning is done with respect to a bounded memory of the factor or with respect to unbounded memory and hence obtain additional information about the magnitude of risk.

Hereafter, we give examples in discrete, absolutely continuous and mixed cases.

4.2.1. Example in a discrete case. Consider the process $\left(X_{i}\right)_{(i \in N)}$ such that $X_{i}=I_{i} \times B_{i}$, where

- $I_{i}$ 's are Bernoulli r.v.'s conditionally to $\underline{V}_{i}$ and conditionally independent with respect to $\underline{V}_{i}$,

- $B_{i}$ 's are considered independent and identically distributed and independent of the $I_{i}$ 's and of $\underline{V}_{i}$. We shall denote by $\mu_{B}$ the common law.

- $\left(V_{i}\right)_{(i \in N)}$ is a mixing sequence of Bernoulli random variables.

This example is inspired from insurance risk theory: if $I_{i}=1$ then there is a claim and $B_{i}$ is the claim amount.

In this example the factor sequence $\left(V_{i}\right)_{i \in \mathbb{N}}$ can modulate the economic environment over time. For example, it could be a Bernoulli random variable, taking value one in time of crisis and value zero in a stable period.

We shall consider that the conditional law of $I_{i}$ has the following structure:

$$
\mathbb{P}\left(I_{i}=1 \mid \underline{V}_{i}\right)=\sum_{j=1}^{i} \frac{h\left(V_{j}\right)}{2^{i-j}},
$$


where $h$ is a measurable, non negative and bounded function: $0<\rho \leq$ $h(v) \leq \frac{\kappa}{2}$, with $\kappa<1$ to ensure that $\mathbb{P}\left(I_{i}=1 \mid \underline{V}_{i}\right)<1$. We have:

$$
\begin{gathered}
\mathbb{P}\left(I_{i}=0 \mid \underline{V}_{i}\right)=\sum_{j=1}^{i} \frac{\xi_{0}^{i}\left(V_{j}\right)}{2^{i-j}} \\
\text { with } \xi_{0}^{i}(v)=\frac{1}{2\left(1-\frac{1}{2^{i}}\right)}-h(v) .
\end{gathered}
$$

Denoting $\xi_{1}^{i}(v)=h(v)$, we may write for $a \in\{0,1\}$

$$
\mathbb{P}\left(I_{i}=a \mid \underline{V}_{i}\right)=\sum_{j=1}^{i} \frac{\xi_{a}^{i}\left(V_{j}\right)}{2^{i-j}} .
$$

Note that $\xi_{a}^{i}$ is bounded, $0<\tau_{1} \leq \xi_{a}^{i}(v) \leq \tau_{2}$. The conditional kernel transition is $N^{i}\left(\cdot \mid \underline{V}_{i}\right)=\delta_{\{0\}} \mathbb{P}\left(I_{i}=0 \mid \underline{V}_{i}\right)+\delta_{\{1\}} \mu_{B} \mathbb{P}\left(I_{i}=0 \mid \underline{V}_{i}\right)$, which rewrites

$$
N^{i}\left(\cdot \mid \underline{V}_{i}\right)=\sum_{a \in\{0,1\}} \delta_{\{a\}} \mu_{B}^{a} \mathbb{P}\left(I_{i}=a \mid \underline{V}_{i}\right)
$$

where $\mu_{B}^{1}=\mu_{B}$ and $\mu_{B}^{0}=1$ and $\delta_{\{.\}}$is the dirac measure.

Lemma 4.2. The transition kernel $N^{i_{1}, \ldots, i_{u}}\left(\cdot \mid \underline{V}_{i_{u}}\right)$ satisfies conditions 2.6 and (2.9) of Assumption 1 .

Proof. We have

$$
\begin{aligned}
N^{i_{1}, \ldots, i_{u}}\left(\cdot \mid \underline{V}_{i_{u}}\right) & =\prod_{k=1}^{u} \sum_{a_{k} \in\{0,1\}} \delta_{a_{k}} \mu_{B}^{a_{k}} \mathbb{P}\left(I_{i_{k}}=a_{k} \mid \underline{V}_{i_{k}}\right) \leq \prod_{k=1}^{u} \sum_{a_{k} \in\{0,1\}} \delta_{a_{k}} \mu_{B}^{a_{k}} \\
& =\sum_{a_{1}, \ldots, a_{u} \in\{0,1\}^{u}} \delta_{a_{1}, \ldots, a_{u}} \mu_{B}^{a_{1}} \cdots \mu_{B}^{a_{k}} .
\end{aligned}
$$

On the other hand,

$$
\begin{aligned}
\left\|N^{i_{1}, \ldots, i_{u}}\left(\cdot \mid \underline{V}_{i_{u}}\right)\right\|_{1} & =\int_{\underline{V}} \prod_{k=1}^{u} \sum_{a_{k} \in\{0,1\}} \delta_{a_{k}} \mu_{B}^{a_{k}} \mathbb{P}\left(I_{i_{k}}=a_{k} \mid \underline{V}_{i_{k}}\right) d \underline{V} \\
& =\sum_{a_{1}, \ldots, a_{u} \in\{0,1\}^{u}} \delta_{a_{1}, \ldots, a_{u}} \mu_{B}^{a_{1}} \cdots \mu_{B}^{a_{k}} \mathbb{P}\left(I_{i_{1}}=a_{1} \ldots I_{i_{u}}=a_{u}\right) .
\end{aligned}
$$

We remark

$$
\begin{aligned}
\mathbb{P}\left(I_{i_{1}}=a_{1} \ldots I_{i_{u}}=a_{u}\right) & =\int_{\underline{V}} \prod_{k=1}^{u} \sum_{j=1}^{i_{k}} \frac{\xi_{a_{k}}^{i_{k}}\left(V_{k}\right)}{2^{i_{k}-j}} d \underline{V} \\
& \geq \tau_{1}^{u} .
\end{aligned}
$$

Finally,

$$
\sup _{\underline{V}} N^{i_{1}, \ldots, i_{u}}\left(\cdot \mid \underline{V}_{i_{u}}\right) \leq\left(\frac{1}{\tau_{1}}\right)^{u}\left\|N^{i_{1}, \ldots, i_{u}}\left(\cdot \mid \underline{V}_{i_{u}}\right)\right\|_{1} .
$$


This proves that condition $(2.9)$ is satisfied.

Now, we consider multi indices such that $i_{1}<\cdots<i_{u}<i_{u}+\ell \leq j_{1}<\cdots<$ $j_{v}$

$$
\begin{aligned}
& D\left(\underline{V}_{j_{v}}, \widetilde{V}_{i_{u}, j_{v}, \ell}\right) \\
= & \sum_{\left(a_{1}, \ldots, a_{v}\right) \in\{0,1\}^{v}} \delta_{a_{1}, \ldots, a_{v}} \mu_{B}^{a_{1}} \times \cdots \times \mu_{B}^{a_{v}} \times \\
& \quad\left[\mathbb{P}\left(I_{j_{1}}=a_{1} \ldots I_{j_{v}}=a_{v} \mid \underline{V}_{j_{v}}\right)-\mathbb{P}\left(I_{j_{1}}=a_{1} \ldots I_{j_{v}}=a_{v} \mid \widetilde{V}_{j_{v}}\right)\right] .
\end{aligned}
$$

Denoting $\widetilde{V}_{j_{v}}=\left(\tilde{V}_{1}, \ldots, \tilde{V}_{j_{v}}\right), \tilde{V}_{j}=0$ for $j=1, \ldots, i_{u}+\ell$ and $\widetilde{V}_{j}=V_{j}$ for $j>i_{u}+\ell$. We have,

$$
\begin{aligned}
\mid & \mathbb{P}\left(I_{j_{1}}=a_{1} \ldots I_{j_{v}}=a_{v} \mid \underline{V}_{j_{v}}\right)-\mathbb{P}\left(I_{j_{1}}=a_{1} \ldots I_{j_{v}}=a_{v} \mid \widetilde{V}_{j_{v}}\right) \mid \\
= & \sum_{\substack{p=1, \ldots, v \\
k_{p}=1, \ldots, j_{p}}} \frac{1}{2^{j_{1}-k_{1}}} \cdots \frac{1}{2^{j_{v}-k_{v}}}\left[\xi_{b_{1}}^{j_{1}}\left(V_{k_{1}}\right) \ldots \xi_{b_{v}}^{j_{v}}\left(V_{k_{v}}\right)-\xi_{b_{1}}^{j_{1}}\left(\tilde{V}_{k_{1}}\right) \ldots \xi_{b_{v}}^{j_{v}}\left(\tilde{V}_{k_{v}}\right)\right] \\
\leq & \left(\begin{array}{l}
v \\
0
\end{array}\right) 2^{v} \sup \mid\left[\xi_{b_{1}}^{j_{1}}\left(V_{k_{1}}\right) \ldots \xi_{b_{v}}^{j_{v}}\left(V_{k_{v}}\right)-\xi_{b_{1}}^{j_{1}}\left(\tilde{v}_{k_{1}}\right) \ldots \xi_{b_{v}}^{j_{v}}\left(\tilde{v}_{k_{v}}\right)\right]+ \\
& \left(\begin{array}{l}
v \\
1
\end{array}\right) \sup \left|\xi_{a}^{i}\right|^{v} \frac{2}{2^{j_{1}-i_{u}-\ell}} \times 2^{v-1}+ \\
& \left(\begin{array}{l}
v \\
2
\end{array}\right) \sup \left|\xi_{a}^{i}\right|^{v} \frac{2^{2}}{2^{j_{1}+j_{2}-2\left(i_{u}+\ell\right)}} \times 2^{v-2}+ \\
& \vdots \\
& \left(\begin{array}{c}
v \\
v-1
\end{array}\right) \sup \left|\xi_{a}^{i}\right|^{v} \frac{2^{v-1}}{2^{\sum_{k=1}^{v-1} j_{k}-(v-1)\left(i_{u}+\ell\right)}} \times 2+ \\
& \left(\begin{array}{l}
v \\
v
\end{array}\right) \sup \left|\xi_{a}^{i}\right|^{v} \sum_{\substack{k_{p} \leq\left(i_{u}+\ell\right) \\
p=1, \ldots, v}} \frac{1}{2^{j_{1}-k_{1}}} \ldots \frac{1}{2^{j_{v}-k_{v}}}
\end{aligned}
$$

and finally,

$$
\begin{aligned}
\left|\mathbb{P}\left(I_{j_{1}}=a_{1} \ldots I_{j_{v}}=a_{v} \mid \underline{V}_{j_{v}}\right)-\mathbb{P}\left(I_{j_{1}}=a_{1} \ldots I_{j_{v}}=a_{v} \mid \widetilde{V}_{j_{v}}\right)\right| & \leq \sum_{i=1}^{v}\left(\begin{array}{l}
v \\
i
\end{array}\right) \frac{\left(2 \tau_{2}\right)^{v}}{2^{i\left(j_{1}-i_{u}-\ell\right)}} \\
& \leq \frac{\left(4 \tau_{2}\right)^{v}}{2^{k}}
\end{aligned}
$$

with $k=j_{1}-i_{u}-\ell$. We have proved

$$
\left|D\left(\underline{V}_{j_{v}}, \widetilde{V}_{i_{u}, j_{v}, \ell}\right)\right| \leq \frac{1}{2^{k}}\left(\frac{4 \tau_{2}}{\tau_{1}}\right)^{v}\left\|N^{i_{1}, \ldots, i_{u}}\left(\cdot \mid \underline{V}_{i_{u}}\right)\right\|_{1},
$$

thus (2.6) is satisfied.

Remark 4. If the function $h$ is not bounded from below, $0<h(v) \leq \frac{\kappa}{2}$, then the functions $\xi_{a}^{i}$ are not bounded from below and (2.6) and (2.9) are not satisfied anymore but (2.7) and (2.10) are satisfied. So that by using Theorem 2.2 we derive mixing properties of the process $\left(X_{i}\right)_{i \in \mathbb{N}}$. 
4.2.2. Example in absolutely continuous case. This subsection is devoted to the case where the conditional law $X_{i} \mid \underline{V}_{i}$ is absolutely continuous. The conditional kernel transition is denoted by $N^{i}\left(d x_{i}, \underline{V}_{i}\right)=f_{\underline{V}_{i}}^{i}(\cdot) d x_{i}$

Example 2. Consider that the conditional law $X_{i} \mid \underline{V}_{i}$ is Pareto $\left(\alpha, \theta_{i}\right)$ where $\alpha>2$ is the shape parameter and $\theta_{i}>0$ is the scale parameter: the conditional density of $X_{i}, i \in \mathbb{N}$, has the form

$$
f_{\underline{V}_{i}}^{i}\left(x_{i} ; \alpha, \theta_{i}\right)=\alpha \times \frac{\theta_{i}^{\alpha}}{x_{i}^{\alpha+1}} \text { for } x_{i} \geq \theta_{i},
$$

where the scale parameter $\theta_{i}$ depends on $\underline{V}_{i}$ with the following structure:

$$
\theta_{i}^{\alpha}=\sum_{j=1}^{i} \frac{h\left(V_{j}\right)}{2^{i-j}}
$$

and where the function $h$ satisfies $0<h(v) \leq \tau_{2}$.

It is interesting to mention that this model has the following useful representation: let $X_{i}$ be distributed as the following conditional distribution given $\underline{V}_{i}$ :

$$
X_{i} \mid \underline{V}_{i}=Z_{i} \times \theta_{i},
$$

where the parameter $\theta_{i}$ is depending on $\underline{V}_{i}$ and where $Z_{i}$ is Pareto $(\alpha, 1)$ distributed and is independent of $\theta_{i}$ then in this case $X_{i} \mid \underline{V}_{i}$ is Pareto $\left(\alpha, \theta_{i}\right)$ distributed.

Remark 5. Conditional Pareto models has been proposed and used in some actuarial contexts in Albrecher et al. (2011)[1 and Constantinescu et al. (2011)[11. In these papers, $X_{i}=\theta_{i} \times Z_{i}$ with $Z_{i}$ as in Example 2 and $\theta_{i}$ being $V_{i}$-measurable. In our framework, this means that the $X_{i}$ 's are conditionally Pareto with respect to $V_{i}$ i.e. the model is with bounded memory with respect to $\left(V_{i}\right)_{i \in \mathbb{N}}$. Our model is more general because it allows long term memory.

If the process $\left(X_{i}\right)_{i \in \mathbb{N}}$ is as in Example 2, the conditional density of $\left(X_{i_{1}}, \ldots, X_{i_{u}}\right) \mid \underline{V}_{i_{u}}$ is

$$
F_{x_{i_{1}}, \ldots, x_{i_{u}}}\left(\underline{V}_{i_{u}}\right)=\frac{\alpha^{u}}{\prod_{k=1}^{u} x_{i_{k}}^{\alpha+1}} \prod_{k=1}^{u} \theta_{i_{k}}^{\alpha} \mathbf{1}_{\left\{x_{i_{k}} \geq \theta_{i_{k}}\right\}} .
$$

Proposition 4.3. If $0<\tau_{1} \leq h(v) \leq \tau_{2}$ for any $v$ then the process of Example 2 satisfies (2.8) and (2.10) of Assumption 1 .

Proof. The fact that 2.10$)$ is satisfied is straightforward from the expression of $F_{x_{i_{1}}, \ldots, x_{i_{u}}}\left(\underline{V}_{i_{u}}\right)$ and the fact that $\tau_{1} \leq h(v) \leq \tau_{2}$ implies: $\tau_{1} \leq\left(\theta_{i}\right)^{\alpha} \leq 2 \tau_{2}$. On one other hand,

$$
\begin{aligned}
& \left|F_{x_{j_{1}}, \ldots, x_{j_{v}}}\left(v_{1}, \ldots, v_{j_{v}}\right)-F_{x_{j_{1}}, \ldots, x_{j_{v}}}\left(0, \ldots, 0, v_{i_{u}+\ell+1}, \ldots, v_{j_{v}}\right)\right| \\
& =\frac{\alpha^{v}}{\prod_{k=1}^{v} x_{j_{k}}^{\alpha+1}}\left|\prod_{k=1}^{v} \theta_{j_{k}}^{\alpha} \mathbf{1}_{\left\{x_{j_{k}} \geq \theta_{j_{k}}\right\}}-\prod_{k=1}^{v} \widetilde{\theta}_{j_{k}}^{\alpha} \mathbf{1}_{\left\{x_{j_{k}} \geq \widetilde{\theta}_{j_{k}}\right\}}\right|
\end{aligned}
$$


where

$$
\widetilde{\theta}_{i}=\sum_{j=1}^{i} \frac{h\left(\widetilde{V}_{j}\right)}{2^{i-j}}
$$

with $\widetilde{V}_{j}=0$ for $j=1, \ldots, i_{u}+\ell$ and $\widetilde{V}_{j}=V_{j}$ for $j \geq i_{u}+\ell+1$. Proceeding as in Subsection 4.2.1, we have that

$$
\left|\prod_{p=1}^{v} \theta_{i_{p}}^{\alpha}-\prod_{p=1}^{v} \widetilde{\theta}_{i_{p}}^{\alpha}\right| \leq \frac{\left(4 \tau_{2}\right)^{v}}{2^{p}}
$$

with $p=j_{1}-i_{u}-\ell$. Also,

$$
\left|\theta_{i}^{\alpha}-\widetilde{\theta}_{i}^{\alpha}\right| \leq \frac{2 \tau_{2}}{2^{p}}
$$

and

$$
\left|\theta_{i}-\widetilde{\theta}_{i}\right| \leq \frac{2 \tau_{2}}{2^{p}} \frac{1}{\alpha \tau_{1}^{\frac{\alpha-1}{\alpha}}}
$$

by applying Taylor's equality. Let us denote by $\Theta_{i}$ the interval $\left[\min \left(\theta_{i}, \widetilde{\theta}_{i}\right), \max \left(\theta_{i}, \widetilde{\theta}_{i}\right)\right]$. We get:

$$
\begin{aligned}
D\left(\underline{V}_{j_{v}}, \widetilde{V}_{i_{u}, j_{v}, \ell}\right) \leq & \frac{\alpha^{v}}{\prod_{k=1}^{v} x_{j_{k}}^{\alpha+1}} \frac{\left(4 \tau_{2}\right)^{v}}{2^{p}} \prod_{k=1}^{v} \mathbf{1}_{\left\{x_{j_{k}} \geq \tau_{1}^{\frac{1}{\alpha}}\right\}} \\
& +\frac{\alpha^{v}}{\prod_{k=1}^{v} x_{j_{k}}^{\alpha+1}}\left(2 \tau_{2}\right)^{v} \prod_{k=1}^{v} \mathbf{1}_{\left\{x_{j_{k}} \in \Theta_{j_{k}}\right\}} .
\end{aligned}
$$

So, we conclude that 2.8 is fulfilled by using that the Lebesgue measure of $\Theta_{i}$ is $\left|\theta_{i}-\widetilde{\theta}_{i}\right|$ and using (4.2) and Fubini's Theorem.

Remark 6. Note that the case of an Exponential conditional law (instead of a Pareto conditional law) could also be considered. In particular if we consider an Exponential law with parameter $\left(1 / \theta_{i}\right)$ where $\theta_{i}$ is defined as in (4.1) with $\alpha=1$ and $0<\tau_{1} \leq h(v) \leq \tau_{2}$ for any $v$, then (2.8) and (2.10) of Assumption 1 are satisfied.

4.2.3. Models for applications of the absolutely continuous case. It is interesting to explain further how our conditionally independent model may have applications in insurance or financial contexts.

Life insurance companies as well as financial institutions use hedging strategies with different portfolio adjustment frequencies: hedging is more frequent for banks, often weekly for life insurance companies offering variable annuities, and more monthly or quarterly for classical life insurance companies, even if some financial lines of business of some insurance companies correspond to financial institutions. All those firms face transaction costs, which prove to be very expensive when things go wrong, both due to liquidity issues and due to other conditions like negative gamma situations. Optimal hedging strategies in presence of liquidity risk and transaction costs have been studied by various authors (see e.g. [28] and references therein). Here, 
we do not aim at studying those optimal strategies; if we rather adopt a P\& C loss model approach, we would try to study the impact of correlations between elementary transaction costs on the aggregated transaction cost. Correlations might come from factors like liquidity and market conditions that vary over time but present some memory. We consider that the aggregated transaction cost is the sum of infinitely many elementary, correlated transaction costs. Assume that $\left(Z_{i}\right)_{i \geq 1}$ is a sequence of i.i.d. random variables representing random transaction costs in a steady environment (without liquidity shocks or bad market conditions), and that the $V_{j}, j \geq 1$ are random variables which contribute to create liquidity shocks and market conditions during some time when they take large values. Consider for each $i \geq 1$,

$$
X_{i}=\theta_{i} . Z_{i},
$$

where

$$
\theta_{i}=\left(\sum_{j=1}^{i} \frac{1}{2^{i-j}} h\left(V_{j}\right)\right)^{1 / \alpha}
$$

corresponds to a multiplicative factor that takes into account liquidity and market conditions at time $i$, the function $h$ is as before, $0<\tau_{1} \leq h(v) \leq \tau_{2}$. The $Z_{i}, i \geq 1$ are independent from the $V_{j}, j \geq 1$. If $Z_{i}$ is $\operatorname{Pareto}(\alpha, 1)$ distributed then $X_{i}$ is Pareto $\left(\alpha, \theta_{i}\right)$ distributed and the $X_{i}$ 's are as in Example 2 ,

If the variables $V_{i}$ are bounded and

$$
\theta_{i}=\left(\sum_{j=1}^{i} \frac{1}{2^{i-j}}\left(1+V_{j}\right)\right)^{1 / \alpha}
$$

then,

$$
\theta_{i+1}^{\alpha}=\frac{1}{2} \theta_{i}^{\alpha}+1+V_{i+1},
$$

which enables one to understand the correlation structure between $\theta_{i}$ and $\theta_{i+1}$, as $V_{i+1}$ is independent from $\theta_{i}$.

As in the previous sections, in that case also, (2.8) and (2.10) of Assumption 1 are satisfied. The use of Theorem 2.2 then leads to strong mixing processes of $\left(X_{i}\right)_{i \in \mathbb{N}}$.

4.2.4. Mixed cases. Other examples that have interest in insurance applications are so called mixed cases where the claim amount $X_{i}$ has the following form

- $X_{i}=I_{i} \times B_{i}$,

- $I_{i}$ 's are Bernoulli r.v.'s conditionally to $\underline{V}_{i}$ and conditionally independent with respect to $\underline{V}_{i}$,

- $B_{i}$ 's are conditionally independent and conditionally independent of the $I_{i}$ 's.

If the $I_{i}$ 's are as in Subsection 4.2.1 and the $B_{i}$ 's are as in Example 2 then following along the lines of the previous examples lead to prove that Conditions (2.8) and (2.10) of Assumption 1 are satisfied. 
Concluding remark. It is well known in the literature that processes satisfying some mixing properties have interesting statistical properties (see [16, 17, 18, 20, 3, 27, 34, 38, and many others) such as moment inequalities, central limit theorem ... The mixing properties satisfied by our models, namely $\left(\eta_{u, v}\right)$-mixing properties, are not standard ones and some more work is needed to get these statistical properties. This is the aim of a forthcoming paper ([29]), in particular, we shall prove a self normalized central limit theorem and study further applications to insurance risk processes.

\section{REFERENCES}

[1] Albrecher, H., Constantinescu, C. and Loisel, S.: Explicit ruin formulas for models with dependence among risks. Insurance: Mathematics and Economics. 2, (2011),265-270. MR 2799308

[2] Asmussen, S.: Risk theory in a markovian environment. Scandinavian Actuarial Journal.2, (1989), 69-100. MR 1035668

[3] Bardet, J. M. Doukhan, P., Lang, G. and Ragache. N.: Dependent Lindeberg central limit theorem and some applications. ESAIM Probab. Stat. 12, (2008), 154-172. MR 2374636

[4] Bernstein, S.N.: Sur l'extension du théorème limite du calcul des probabilités aux sommes de quantités dépendantes. Math. Ann. (1927) 97, 1-59.

[5] Billingsley, P.: Convergence of probability measures. Wiley. (1968). MR 1700749

[6] Blum, J. R., Hanson, D.L. and Koopmans., L. H.: On the strong law of large number for a class of stochastic processes. Z. Wahrscheinlichkeitstheorie und Verw. Gebiete. 2, (1963), 1-11. MR 0161369

[7] Bradley, R.C. and Bryc, W.: Multilinear forms and measures of dependence between random variables. Journal of Multivariate Analysis. 16, (1985), 335-367. MR 0793497

[8] Bradley, R.C.: Basic properties of strong mixing conditions. Dependence in probability and statistics.Progr. Probab. Statist. 11, (1986), 165-192. MR 0899990

[9] Bradley, R.C.: Basic properties of strong mixing conditions. A survey and some open questions. Update of, and a supplement to, the 1986 original. Probability Surveys. 2,(2005), 107-144. MR 2178042

[10] Bradley, R.C.: Introduction to Strong Mixing Conditions. Kendrick Press, Heber City, Utah. 1,2,3, (2007). MR 2325294, MR 2325295, MR 2325296

[11] Constantinescu, C., Hashorva, E. and Ji L.: Archimedean copulas in finite and infinite dimensions - with application to ruin problems. Insurance: Mathematics 85 Economics. 49,(2011), 487-495.

[12] Cossette, H., Gaillardetz, P. and Marceau, E.:Common mixture in the individual risk model. Mitteilungen der Schweiz. Aktuarvereinigung. 2, (2002), 131-157. MR 1908350

[13] Cossette, H., David, L. and Marceau, E.: Compound binomial risk model in a markovian environment. Insurance: Mathematics \& Economics. 35 (2004), 425-443. MR 2095897

[14] Coulon-Prieur, C. and Doukhan, P.: A triangular central limit theorem under a new weak dependence condition. Statistics \& Probability Letters. 47,(2000), 61-68. MR 1745670

[15] Davydov, YU. A.: The Convergence of distributions generated by stationary stochastic processes. Theory of Probability and Its Application. 13,(1968), 691-696.

[16] Dedecker, J. and Doukhan, P.: A new covariance inequality and applications. Stochastic Process. Appl. 106 (2003), 63-80. MR 1983043

[17] Dedecker, J., Doukhan, P., Lang, G., León R, J. R., Louhichi, S. and Prieur, C.: Weak Dependence: With Examples and Applications. Lecture Notes in Statistics, 190. Springer, New York. (2007). MR 2338725

[18] Dedecker, J. and Prieur, C.: New dependence coefficients. Examples and applications to statistics. Prob. Th. Rel. Fields. 132 (2005), 203-236. MR 2199291

[19] Doukhan, P.: Mixing properties and examples. Lecture note in statistics. 85. (1994). MR 1312160. 
[20] Doukhan, P. and Louhichi S.: A new weak dependence condition and applications to moment inequalities. Stochastic Processes and their Applications. 84, (1999), 313342. MR 1719345

[21] Dziubdziela, W.: Limit laws for $k$ th order statistics from conditionally mixing arrays of random variables. Journal of Applied Probability. 23, (1986), 679-687. MR 0855374

[22] Gerber, H. U.: Mathematical fun with the compound binomial process. ASTIN Bull. 18, (1988), 161-168.

[23] Gordin, M. I.: On the central limit theorem for stationary random processes. Dokl. Akad. Nauk. SSSR.(1969), 188, 739-741. MR 0251785

[24] Gordin, M. I.: A note on the martingale method of proving the central limit theorem for stationary sequences. Journal of Mathematical Sciences. 133, (2006). MR 2092203

[25] Hall, P. and Heyde, C. C.: Martingale limit theory and its application. Probability and Mathematical Statistics. Academic Press, Inc,New York-London. (1980). MR 0624435

[26] Ibragimov, I.A.: Some limit theorems for stationary processes. Theory of Probability and its Applications. 7, (1962), 349-382.

[27] Ibragimov, I.A. and Linnik, Y. U.: Independent and stationary sequences of random variables. Wolters-Noordhoff Publishing, Groningen. (1971), 443. MR 0322926

[28] Kabanov, Y. M.: Hedging and liquidation under transaction costs in currency markets. Finance and Stochastics. 2, (2009), 237-248.

[29] Kacem, M., Loisel, S. and Maume-Deschamps V.: Self normalized central limit theorems for conditionally independent variables and applications to risk processes. Work in progress.

[30] Kolmogorov, A. N. and Rozanov, Ju. A.: On a strong mixing condition for stationary Gaussian processes. Theory Of Probability and Its Applications. V, (1960), 204-208. MR 0133175

[31] Mcleish, D. L.: Invariance principle for dependent variables. Z. Wahrscheinlichkeitstheorie und Verw. Gebiete 32,(1975) 165-178. MR 0388483

[32] Merlevède, F., Peligrad, M. and Utev, S.: Recent advances in invariance principles for stationary sequences. Probability Surveys. (2006), 3, 1-36. MR 2206313

[33] Obrein, G.L.: Limit theorems for sums of chain-dependent processes. Journal of applied probability. 11, (1974), 582-587. MR 0368159

[34] Peligrad, M.: Recent advances in the central limit theorem and its weak invariance principle for mixing sequences of random variables. Dependence in probability and statistics (Oberwolfach,1985). Progress Probab. Statist. (1986), 11, 193-223. MR 899991

[35] Philipp, W. and Stout, W.F.: Almost sure invariance principles for partial sums of weakly dependent random variables. Memoirs of the American Mathematical Society. 2, (1975), iv+140. MR 0433597

[36] Rao, P.: Conditional independence, conditional mixing and conditional association. Annals of the Institute of Statistical Mathematics (2009), 61, 441-460. MR 2505397

[37] Rio, E.: Covariance inequalities for strongly mixing processes. Ann. Inst. H. Poincaré Probab. Statist. 29, (1993), 587-597. MR 1251142

[38] Rio, E.: Inégalités de moments pour les suites stationnaires et fortement mélangeantes. C. R. Acad. Sci. Paris Sér. I Math. 318,(1994), 355-360. MR 1267615

[39] Rosenblatt, M.: A central limit theorem and a strong mixing condition. Proceeding national academy of Sciences of the United States of America. (1956), 42, 427-433. MR 0074711

[40] Veijanen, A.: On conditionally mixing processes. Statistics $\&$ Probability Letters. 9, (1990), 59-65. MR 1035992

Université de Lyon, Université Lyon 1, ISFA, Laboratoire SAF EA 2429

E-mail address: manel.kacem@univ-lyon1.fr

Université de Lyon, Université Lyon 1, ISFA, LABoratoire SAF EA 2429

E-mail address: stephane.loisel@univ-lyon1.fr

Université De Lyon, Université Lyon 1, ISFA, Laboratoire SAF EA 2429

E-mail address: veronique.maume@univ-lyon1.fr 